\title{
ON THE FIELD ANOMALY OF NEAR WAKES IN A COLLISIONLESS PLASMA*
}

\author{
V.C. LIU and H. JEW \\ The University of Michigan, Ann Arbor, Michigan, USA
}

Received 3 November 1972

Revised manuscript received 22 January 1973

\begin{abstract}
A variational approach is proposed to determine the induced electric field by charge separation in the near wake of a large negatively charged body moving at mesothermal speeds in a tenuous plasma. The presence of potential well is discussed.
\end{abstract}

The interaction between the ionospheric medium and a rapidly moving body provides a unique plasma phenomenon of much geophysical consequences [1]. The moving body in question, e.g., a space probe or meteorite, can be charaterized by the following conditions: $\lambda_{\mathrm{D}} \ll R \ll l ; c_{\mathrm{i}} \ll V \ll c_{\mathrm{e}}$ where $R$ and $V$ denote the size and the speed respectively of the body; $\lambda_{\mathrm{D}}, l$, $c_{\mathrm{i}}$ and $c_{\mathrm{e}}$, the Debye length, mean free path, ion thermal speed and electron thermal speed respectively of the plasma. It is well known that when a neutral plasma meets a moving blunt body a potential sheath having a thickness of the order $\lambda_{D}$ develops to shield the frontal body. In the near wake behind a rapidly moving body where the electrons lead the ions in filling the void region, a charge separation field in the form of a potential valley is created. In other words, the potential distribution in the near wake, instead of varying monotonically from its boundary value at the body surface $\phi_{S}$ (usually $<0$ ) to zero at the free stream, dips into a well of depth $\left|\phi_{\min }\right|>\left|\phi_{\mathrm{s}}\right|$ before it rises to its ambient value (zero). This anomalous field configuration which has been shown to cause plasma trapping [2] and possibly other consequences yet to be explored is of interest in the present note.

Consider a conducting spherical body of radius $R$ and surface potential $\phi_{\mathrm{S}}(<0)$ which moves at a steady mesothermal speed $V$ in a bithermal neutral plasma of singly charged ions at temperature $T_{\mathrm{i}}$ and free electrons at temperature $T_{\mathrm{e}}$ where $\lambda_{\mathrm{D}} \ll R \ll l$. It is assumed that in collision with the body an electron is absorbed; an ion, neutralized and re-emitted as a neutral particle. The magnetic field effect is neglected. These conditions hold

\footnotetext{
* Supported jointly by NASA Grant NGR 23-005-094 and NGR 23-005-320.
}

e.g., for a typical satellite-motion in the upper ionosphere [3]. In the following analysis the origin of a cylindrical coordinate is fixed to the center of the moving body with its $z$-axis aligned with the axis of the wake. The linear displacement $r$ is normalized by $R$; field potential $\phi(r)$ by $e / k T_{\mathrm{i}}$ where $e$ and $k$ denote the electronic charge and Boltzmann constant respectively; particle velocity $c$ by $c_{\mathrm{i}}$ and the particle density $n(\boldsymbol{r})$ by $n_{\infty}$, the free stream electron or ion density. The conditions $\phi_{\mathrm{s}}<0$ and $V \ll c_{\mathrm{e}}$ imply that the electron distribution in the wake will deviate from its free stream value only by the Boltzmann factor thus $n_{\mathrm{e}}(r)=\exp \left(\phi T_{\mathrm{i}} / T_{\mathrm{e}}\right)$. On the other hand since $V \gg c_{\mathrm{i}}$ the ion distribution $f(r, c)$ in the wake will differ strongly with its free stream equilibrium state. In fact $f(r, c)$ and $\phi(r)$ are governed by the following coupled equations of the collision free (particle) equation and the Poisson (field) equation [3]:

$c \cdot \nabla_{r} f-\frac{1}{2}\left(\nabla_{r} \phi\right) \cdot \nabla_{c} f=0 ;\left(\lambda_{\mathrm{D}} / R\right)^{2} \nabla_{r}^{2} \phi=n_{\mathrm{i}}-n_{\mathrm{e}}$ where $n_{\mathrm{i}}=\int f \mathrm{~d} c$ with $\phi=\phi_{\mathrm{s}}$ at $r=R$ and $\phi=0$ at $r \rightarrow \infty$.

An iterative numerical scheme has often been used [3] to treat the nonlinear system (1). It starts with a formal solution for $f(r, c)$ from the quasi-linear first order particle equation, in terms of the appropriate invariants. Notice that an analogous mathematical formalism in stellar dynamics is known as Jeans theorem [4]. For an axi-symmetric field herein interested three isolating invariants are needed. The energy invariant $E=c_{\rho}^{2}+c_{\theta}^{2}+c_{z}^{2}+\phi$ and angular momentum invariant $L_{z}=|r \times c|_{z}$ are obvious [3]; the existence of the third invariant for a general arbitrary axi-symmetric $\phi(r)$, however, has not been settled. Nonetheless, if the potential is prescribed as $\phi_{\mathrm{L}}=\zeta(r)+\eta(\theta) / r^{2}$ where $\zeta(r)$ and 
$\eta(\theta)$ are arbitrary functions of the radius vector $r$ and the polar angle $\theta$ respectively, it is known that the third invariant $I_{\mathrm{L}}=|\boldsymbol{r} \times c|^{2}+\eta(\theta)[4]$.

It is suggested herein to use a variational method that yields approximate solutions to the system (1). It consists of finding a functional $J(\phi, f)$ whose variation gives the equations (1) as its "Euler equations" [5].This is found to have the following form:

$$
\begin{gathered}
J(\phi, f)=\frac{1}{2}\left(\lambda_{\mathrm{D}} / R\right)^{2}\left\{\int(\nabla \phi)^{2} \mathrm{~d} r+\phi_{\mathrm{s}} \int_{\text {sphere }}[\partial \phi(1, \theta) / \partial r] \mathrm{d} \theta\right\} \\
-\iint\left(n_{\mathrm{i}}-n_{\mathrm{e}}\right) \mathrm{d} \phi^{\prime} \mathrm{d} r+\iint\left[c \cdot \nabla_{r} f+\left(\nabla_{r} \phi\right) \cdot \nabla_{c} f\right] f^{\prime} \mathrm{d} c \mathrm{~d} \boldsymbol{r}
\end{gathered}
$$

for an axi-symmetric wake. Trial functions for $\phi(r)$ and $f(r, c)$ must be furnished to functional (2) before a variational principle can be applied. It is intended to introduce a perturbed pair $\left(\phi, I_{3}\right)$ which is related to the unperturbed $\left(\phi_{\mathbf{L}}, I_{\mathrm{L}}\right)$-pair as follows * [5]:

$\phi=\phi_{\mathrm{L}}+\eta_{1}(r, \theta) / r^{2}, \quad I_{3}=I_{\mathrm{L}}+\eta_{1}(r, \theta)$

$I_{3}$ will be used along with $E$ and $L_{z}$ to prescribe the trial $f(r, c)=f\left(E, L_{z}, I_{3}\right)$ following Jeans theorem. In compliance with (3), the suggested trial potential $\phi(r)$ which satisfies the wake boundary conditions is prescribed as follows:

$$
\begin{aligned}
\phi= & \left\{\phi_{\mathrm{s}}-(r-1) \cos ^{2} \theta\left[B_{0} p_{0}+r B_{1} p_{1} \exp -(r-1)\right.\right. \\
+ & r^{2} B_{2} p_{2} \exp -2(r-1) \\
& \left.\left.+r^{i} B_{i} p_{i} \exp i(r-1)+\ldots\right]\right\} \exp -(r-1)
\end{aligned}
$$

and its associated third invariant:

$$
\begin{aligned}
I_{3}= & r^{2}\left(c_{\theta}^{2}+c_{\phi}^{2}\right)-r^{2}(r-1) \cos ^{2} \theta\left[B_{0}+\right. \\
+ & r B_{1} p_{1} \exp -(r-1)+r^{2} B_{2} p_{2} \exp -2(r-1) \\
& \left.+r^{i} B_{i} p_{i} \exp -i(r-1)+\ldots\right] \exp -(r-1)
\end{aligned}
$$

where $p_{i}(i=0,1,2,3, \ldots)$ are Legendre polynomials; $B_{i}(i=0,1,2, \ldots)$, coefficients to be determined with variational method. The trial $f\left(E, L_{z}, I_{3}\right)$ satisfying its boundary conditions [3] can be written

$f=\frac{1}{2} \pi^{-3 / 2}[1+\operatorname{sign}(S-1)] \exp \left[-\left(E-V^{2}\right)\right]$

* A priori restriction thus implied is that $\phi$ deviates only slightly from $\phi_{\mathbf{L}}$.

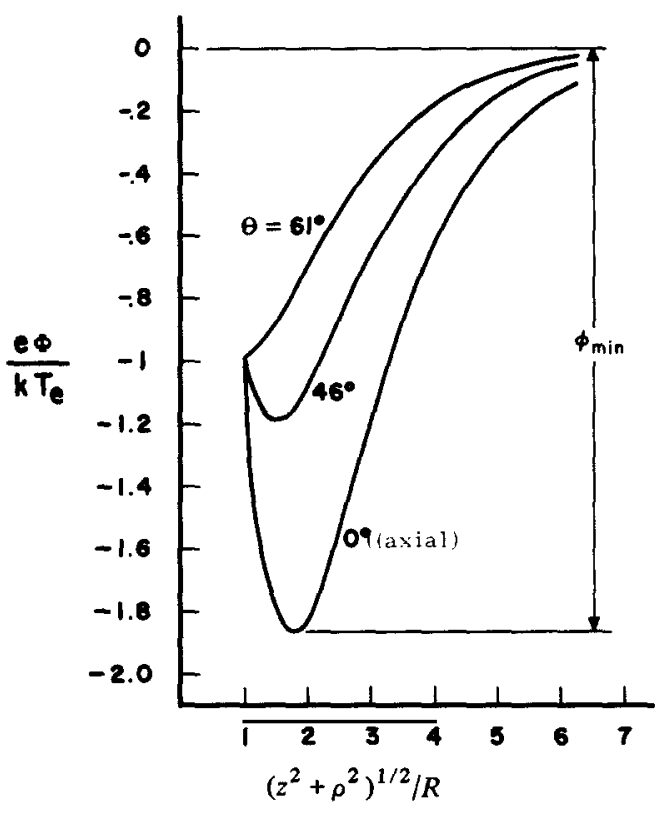

Fig. 1. Electric potential distribution in an axi-sy mmetric wake. where $S=I_{3} / I_{3}$ (evaluated at $\theta=\pi / 2$ ). The vanishing of the differential coefficients $\partial J / \partial B_{i}$ in the first variant $\delta J$, after the substitution of eqs. (4), (5), and (6) in the functional (2), yields a set of nonlinear transcendental algebraic equations which can be solved by the generalized Newton's method. The result, $\phi(r)$, of a case with $\phi_{\mathrm{S}}=-1, V / c_{\mathrm{i}}=8, \lambda_{\mathrm{D}} / R=0.05$ and $T_{\mathrm{e}}=T_{\mathrm{i}}$ is illustrated in fig. 1 . In this illustrated variational calculation, the terms byond $B_{3}$ and $p_{3}$ in eqs. (4) and (5) have been truncated. Calculations including the $B_{4}$ and $p_{4}$ terms have also been made with, however, negligible change in results of $\phi(r)$ and $n_{\mathrm{i}}(\boldsymbol{r})$. The present result (fig. 1) agrees well with previous numerical iteration results in values $\phi_{\min }$ but shows a slight shift in $\phi$-distribution [3]. The locus of the potential minimum $\left(\phi_{\min }\right)$ forms a conical surface in an axi-symmetric wake. The presence of a potential valley in the wake stems from the charge separation as the ambient electrons and ions move into the wake with unequal mass motions.

[1] V.C. Liu, On interplanetary gas dynamics. Adv. App. Mech. 12 (Acad. Press 1972) p. 195.

[2] V.C. Liu, Nature 215 (1967) 127.

[3] V.C. Liu, Space Sci. Rev, 9 (1969) 423.

'[4] D. Lynden-Bell, M.N. Royal Astron. Soc. 124 (1962) 1, 95.

[5] M. Becker, The principles and applications of variational methods (MIT Press, 1964). 